\title{
Monte Carlo Functional Expansion Calculation of Free Molecular Flows*
}

\author{
By A. G. Petschek**, R. E. Williamson, W. J. Krauser and P. C. White \\ Abstract. A Monte Carlo technique for Knudsen flow, light transmission in tubes, \\ the flow of cold neutrons, phonon transmission, heat transfer calculations and other \\ problems involving free streaming in cylindrical enclosures is described. The coeffi- \\ cients of a functional expansion of the view factors are estimated by Monte Carlo, \\ are then perturbed in a least squares minimum sense to satisfy some consistency \\ conditions, and finally are used to calculate the collision density on the boundary \\ and the transmission of the enclosure. Without importance sampling or other vari- \\ ance reduction techniques, the calculational work required for a given accuracy is \\ reduced by a factor frequently near 10 when compared to a simple calculation in \\ which particles are followed from entrance to exit.
}

1. Introduction. We have developed a novel Monte Carlo (MC) technique suitable for application to Knudsen flow, heat transfer, and allied problems. In this technique the coefficients of a polynomial expansion of the collision density are estimated by a stochastic process. Compared to the technique introduced by Davis [1] our code gives comparable accuracy with a considerable saving in computer time. A preliminary report on this method has appeared earlier [2].

Monte Carlo techniques have long been applied to streaming in pipes. For example, Davis [1] and Garelis and Wainwright [3] have applied it to free molecular flow, Fleck [4] to photon flow in a superradiant laser rod, Blechschmidt [5] to light transmission in a cylindrical tube and Berceanu [6] to the flow of cold neutrons. The calculation of view factors in heat transfer problems [7] is also amenable to MC estimation and the heat flow resulting can be determined as we shall describe.

In Davis' technique, particles are started at the source end of the tube (which may have various bends and obstructions in it) and pursued by MC until they emerge at one end or the other. The transmission of the tube is estimated from the number of particles emerging from the far end. This procedure appeared to us to have the disadvantage that, unless sophisticated weighting techniques are used, the far end of the tube is not well explored and large statistical errors result. As an alternative, one might imagine cutting the tube into zones and estimating by MC the view factors, i.e., the probability that a molecule emitted by one zone will impact on another. These

Received April 28, 1978; revised August 28, 1978.

AMS (MOS) subject classifications (1970). Primary $65 \mathrm{C05}$.

*This work performed under the auspices of USDOE, University of California, Los Alamos Scientific Laboratory.

** Consultant permanent address: New Mexico Institute of Mining and Technology, Socorro, New Mexico 87801. 
view factors can then be used in a deterministic calculation of the transfer problem. This method suffers from the disadvantage that many zones must be taken to give a good representation of the collision density. The large number of zones means particles cross many boundaries and adds to the labor of the MC estimates.

Polynomial expansions have been used with MC estimates of integrals [8] and have been suggested for use in shielding [9] and electron transport [10] calculations. We extend these notions to make a MC estimate of a double polynomial expansion of view factors in both the point of origin and the point of impact.

The following sections describe the basic MC loop, the method by which the resulting coefficients are made consistent with reciprocity and conservation requirements, and the use of these stochastically determined coefficients in a calculation of collision densities and transmission. It can be seen that this calculation leads to a small bias which we estimate. To reduce this bias, we propose the use of a method akin to Richardson extrapolation. Finally, we show our results and compare our calculational labor to that in the Davis technique.

The geometries to which we apply the technique have cylindrical symmetry throughout, but we do not believe this limitation is inherent in the method. As we show later, for cylindrical symmetry, most of the improvement in accuracy is obtained when the second polynomial (i.e. linear) term is included. Hence, in many geometries without cylindrical symmetry we would expect to require only three or four harmonics to obtain comparably good results. Functions that might be used for pipes with slight bends, for example, are products of Legendre polynomials along the axis and trigonometric functions in angle. For ducts of rectangular cross section we would treat each plane as a surface and use products of Legendre polynomials or trigonometric functions.

2. Monte Carlo. In a cylindrically symmetric tube of variable cross section one coordinate suffices to describe a position on the tube surface. We have divided the walls of the tube into several zones or subsurfaces. Since no ambiguity should arise, we denote both the $k$ th subsurface and its surface area by $S_{k}$. On each such subsurface, we define a coordinate $a_{k}$. Ordinarily, we choose $a_{k}$ to be the cumulative area along $S_{k}$ from one end to the point considered, with values $a_{k}=0$ and $a_{k}=S_{k}$ at the two ends of the subsurface. The collision density $\theta_{k}$ on each subsurface is expanded in a set of orthogonal polynomials as follows

$$
\theta_{k}\left(a_{k}\right)=\sum_{r} \theta_{k}^{r} p_{k}^{r}\left(a_{k}\right)
$$

Ordinarily, we choose $p_{k}^{r}$ by

$$
p_{k}^{r}\left(a_{k}\right)=\sqrt{2 r+1} P_{k}^{r}\left(\frac{2 a_{k}}{S_{k}}-1\right),
$$

where $P_{k}^{r}$ is the $r$ th ordinary Legendre polynomial, and the index $k$ refers to the subsurface. In principle, of course, the sets of polynomials $P_{k}^{r}$ may be different for different $k$, as may the definitions of the $a_{k}$.

The differential view factor $\gamma_{k l}\left(a_{k}, a_{l}\right)$, which represents the probability density 
that a particle leaving $d a_{l}$ on $S_{l}$ will impact $d a_{k}$ on $S_{k}$, is expanded in a double polynomial series

$$
\gamma_{k l}\left(a_{k}, a_{l}\right)=\sum_{r, s} M_{k l}^{r s} \sqrt{\frac{(2 r+1)(2 s+1)}{S_{k} S_{l}}} P_{k}^{r}\left(\frac{2 a_{k}}{S_{k}}-1\right) P_{l}^{s}\left(\frac{2 a_{l}}{S_{l}}-1\right)
$$

The values of $M_{k l}^{r s}$ are to be estimated by Monte Carlo. Inversion of Eq. (3) gives

(4) $M_{k l}^{r s}=\sqrt{\frac{(2 r+1)(2 s+1)}{S_{k} S_{l}}} \int_{S_{k}} d a_{k} \int_{S_{l}} d a_{l} P_{k}^{r}\left(\frac{2 a_{k}}{S_{k}}-1\right) P_{l}^{s}\left(\frac{2 a_{l}}{S_{l}}-1\right) \gamma_{k l}\left(a_{k}, a_{l}\right)$.

To evaluate this integral we start from each subsurface $N_{0}$ particles with weight uniformly distributed in $a_{l}$, headed in directions determined by the cosine distribution (diffuse reflection). Once the point of first collision of the $i$ th particle is determined to be, say $a_{k}^{(i)}$ on $S_{k}$, the quantity

$$
P_{k}^{r}\left(\frac{2 a_{k}^{(i)}}{S_{k}}-1\right) P_{l}^{s}\left(\frac{2 a_{l}^{(i)}}{S_{l}}-1\right)
$$

is added to tallies proportional to $\hat{M}_{k l}^{r s}$ for all orders $r$ and $s$ less than some cutoff (typically set at 5). Since $P^{0}$ and $P^{1}$ are at hand, and recursive calculation of higher order polynomials is rapid, the tallying adds little to the total running time. The squares of the contributions are tallied as well. When $N_{0}$ particles have been run, we have estimates of the finite area view factors (as given by Eq. (4)) and the associated variances for one particular $l$ and all $k, r$, and $s$.

3. Adjustment of Coefficients. Consider a lossless system with a vacuum interior. In equilibrium the system must satisfy the requirement of detailed balance; i.e., equal numbers of particles must be exchanged between any infinitesimal pair of wall regions. Conservation requires that each particle emitted by a wall region must eventually strike either the same wall region or some other wall region.

By definition, detailed balance gives us

$$
\gamma_{k l}\left(a_{k}, a_{l}\right) d a_{k} d a_{l}=\gamma_{l k}\left(a_{l}, a_{k}\right) d a_{l} d a_{k}
$$

and conservation gives us

$$
\sum_{k} \int_{S_{k}} d a_{k} \gamma_{k l}\left(a_{k}, a_{l}\right)=1
$$

Substitution of (5) into (4) gives, by inspection,

$$
M_{k l}^{r s}=M_{l k}^{s r},
$$

which is the condition for detailed balance. From (4) we have

$$
M_{k l}^{0 s}=\sqrt{\frac{2 s+1}{S_{k} S_{l}}} \int_{S_{k}} d a_{k} \int_{S_{l}} d a_{l} P_{k}^{0}\left(\frac{2 a_{k}}{S_{k}}-1\right) P_{l}^{s}\left(\frac{2 a_{l}}{S_{l}}-1\right) \gamma_{k l}\left(a_{k}, a_{l}\right) .
$$

Using $P_{k}^{0}=1$, Eq. (6), and 


$$
\int_{S_{l}} \sqrt{2 s+1} P_{l}^{s}\left(\frac{2 a_{l}}{S_{l}}-1\right) d a_{l}=\delta_{s 0} S_{l}
$$

gives

$$
\sum_{k} \sqrt{\frac{S_{k}}{S_{l}}} M_{k l}^{0 s}=\delta_{0 s}
$$

The Monte Carlo generated view factors $\hat{M}_{k l}^{r s}$, in general, satisfy neither (7) nor, unless $s=0$, (8). Thus, the direct use of the $\hat{M}_{k l}^{r s}$ in an equilibrium flow calculation results in a nonuniform collision density and nonconservation of particles. Furthermore, we find the accuracy of the transmission estimate in a flow calculation to be poor when the $\hat{M}_{k l}^{r s}$ are used directly. In one case we found the standard deviation of the result to be four times the standard deviation of a calculation using adjusted coefficients (the adjustment procedure is described shortly), and there are biases of about 8 standard deviations and opposite sign at the two ends of the pipe leading to the loss of $1.017 \pm 0.002$ particles for each particle entering. Therefore, we find it highly desirable to adjust the $\hat{M}_{k l}^{r s}$ (in a least squares sense, for instance) so that the modified values $M_{k l}^{r s}$ do satisfy (7) and (8).

Thus, we might seek to minimize

$$
Q^{\prime}=\sum_{k, l, r, s}\left(M_{k l}^{r s}-\hat{M}_{k l}^{r s}\right)^{2},
$$

subject to the constraints (7) and (8). However, the Monte Carlo estimates $\hat{M}_{k l}^{r s}$ generally have a considerable variation in precision, so in minimizing $Q^{\prime}$, we do not make use of all the available information. To put the adjusted moments $M_{k l}^{r s}$ on an equal basis, we choose instead to minimize

$$
Q=\sum_{k, l, r, s} \frac{\left(M_{k l}^{r s}-\hat{M}_{k l}^{r s}\right)^{2}}{\hat{\omega}_{k l}^{r s}}
$$

where $\hat{\omega}_{k l}^{r s}$ is the Monte Carlo estimate of the variance of $\hat{M}_{k l}^{r s}$.

If we have $N$ subsurfaces and $P+1$ moments, the constraint equations reduce to

$$
\begin{aligned}
M_{k l}^{r s}-M_{l k}^{s r}=0, & k=1, \ldots, N ; l=1, \ldots, k ; \\
& \text { and, if } l=k: r=1, \ldots, P ; s=0, \ldots, r-1
\end{aligned}
$$

(Lagrange multiplier $=-X_{k}^{r s}$ )

but, if $l<k: r=0, \ldots, P$; $s=0, \ldots, P$

(Lagrange multiplier $=-Y_{k l}^{r s}$ ),

and

$$
\sum_{k} \sqrt{\frac{S_{k}}{S_{l}}} M_{k l}^{0 s}-\delta_{s 0}=0, \quad l=1, \ldots, N ; s=0, \ldots, P
$$

(Lagrange multiplier $\left.=-V_{l}\right)$.

Using the method of Lagrange multipliers, we seek the minimum of 


$$
\begin{aligned}
Z \equiv & \frac{1}{2} \sum_{k, l, r, s}\left(M_{k l}^{r s}-\hat{M}_{k l}^{r s}\right)^{2} / \hat{\omega}_{k l}^{r s}-\sum_{l=1}^{N} \sum_{s=0}^{P} V_{l}^{s}\left(\sum_{k=1}^{N} \sqrt{\frac{S_{k}}{S_{l}}} M_{k l}^{0 s}-\delta_{s 0}\right) \\
& -\sum_{k=1}^{N} \sum_{r=1}^{P} \sum_{s=0}^{r-1} X_{k}^{r s}\left(M_{k k}^{r s}-M_{k k}^{s r}\right)-\sum_{k=2}^{N} \sum_{l=1}^{k-1} \sum_{r=0}^{P} \sum_{s=0}^{P} Y_{k l}^{r s}\left(M_{k l}^{r s}-M_{l k}^{s r}\right) .
\end{aligned}
$$

At the minimum

$$
\frac{\partial Z}{\partial M_{k l}^{r s}}=\frac{M_{k l}^{r s}-\hat{M}_{k l}^{r s}}{\hat{\omega}_{k l}^{r s}}-U_{k l}^{r s}-V_{l}^{s} \sqrt{\frac{S_{k}}{S_{l}}} \delta_{r 0}=0
$$

where

$$
U_{k l}^{r s}= \begin{cases}Y_{k l}^{r s}, & l<k, \\ X_{k}^{r s}, & l=k, s<r, \\ 0, & l=k, s=r, \\ -X_{k}^{s r}, & l=k, s>r, \\ -Y_{l k}^{s r}, & l>k .\end{cases}
$$

Solve (9) for $M_{k l}^{r s}$ :

$$
M_{k l}^{r s}=\hat{\omega}_{k l}^{r s}\left(U_{k l}^{r s}+V_{l}^{s} \delta_{r 0} \sqrt{S_{k} / S_{l}}\right)+\hat{M}_{k l}^{r s}
$$

Note that we are done when we have solved for $U_{k l}^{r s}$ and $V_{l}^{s}$. Substitute (10) into (7)

$$
\hat{\omega}_{k l}^{r s}\left(U_{k l}^{r s}+V_{l}^{s} \delta_{r 0} \sqrt{S_{k} / S_{l}}\right)+\hat{M}_{k l}^{r s}=\hat{\omega}_{l k}^{s r}\left(U_{l k}^{s r}+V_{k}^{r} \delta_{s 0} \sqrt{S_{l} / S_{k}}\right)+\hat{M}_{l k}^{s r} .
$$

But $U_{k l}^{r s}=-U_{l k}^{s r}$, so we get

$$
U_{k l}^{r s}=\frac{1}{\hat{\omega}_{k l}^{r s}+\hat{\omega}_{l k}^{s r}}\left(\hat{M}_{l k}^{s r}-\hat{M}_{k l}^{r s}+\hat{\omega}_{l k}^{s r} \delta_{s 0} V_{k}^{r} \sqrt{\frac{S_{l}}{S_{k}}}-\hat{\omega}_{k l}^{r s} \delta_{r 0} V_{l}^{s} \sqrt{\frac{S_{k}}{S_{l}}}\right)
$$

Now we are done when we have solved for $V_{k}^{r}$. Substitute (10) into (8):

$$
\sum_{k} \sqrt{\frac{S_{k}}{S_{l}}}\left[\hat{\omega}_{k l}^{0 s}\left(U_{k l}^{0 s}+V_{l}^{s} \sqrt{S_{k} / S_{l}}\right)+\hat{M}_{k l}^{0 s}\right]=\delta_{s 0} .
$$

Substituting (11) into (12) gives $N \cdot(P+1)$ linear equations $(l=1, \ldots, N$ and $s=$ $0, \ldots, P)$ in $N \cdot(P+1)$ unknowns $\left(V_{l}^{S}\right)$, and machine solution is straightforward.

The use of variances correlated with the estimates of $M$ leads to a bias because the weight assigned to a particular $\hat{M}$ on a run on which that $\hat{M}$ happens to be small is larger than the weight assigned in a run in which it happens to be large. In the simple case of two equal surfaces only weakly coupled to each other, one can see that the result of using variances from the same run to estimate $M_{k l}^{00}$ gives the geometric rather than the arithmetic mean of the number of hits. With good statistics (large $N_{0}$ ) 
this bias becomes small. We have not found the bias significant, but it appears wiser to take $\hat{M}$ and $\hat{\omega}$ for different MC runs and average later. We have been unable to find a suitable unbiased set of weights not derived from a MC run.

Occasionally, furthermore, no particles happen to have gone from one zone to another, and this leads to an infinite weight for the associated $M$ s. We have generally modified this large weight one way or another, but this seems not to have a significant effect on the results.

It is often the case that the Monte Carlo particles are started with uniform probability in $a_{l}$ and unit weight. Under these conditions, the coefficients $\hat{M}_{k l}^{00}$ are proportional to the fraction of particles emitted by $S_{l}$ which subsequently strike $S_{k}$ on first collision. Thus, these coefficients are multinomially distributed which would allow us to use the method of maximum likelihood rather than the method of least squares in the adjustment procedure. One could, therefore, improve the coefficient adjustment scheme by using a hybrid procedure whereby the $\hat{M}_{k l}^{00}$ are treated in a manner that utilizes the known form of the distribution function, and the $\hat{M}_{k l}^{r s}(r, s>0)$ are treated by the least squares method described earlier. The adjustment equations have been derived and do admit a solution. As before, of course, the adjusted coefficients would be biased.

4. The Flow Problem. We assume that the surfaces $S_{k}$ completely define the boundaries of the enclosure of interest and fall into two classes, for one of which (called source and sink surfaces) the total particle emission rate $\theta_{k}\left(a_{k}\right)$ is known while the fluxes $\varphi_{k}\left(a_{k}\right)$ are not, whereas for the other class of surfaces, $\theta$ is to be calculated while the fluxes either vanish for a lossless system, or are proportional to the emission rate $\theta$ for a partly reflecting surface.

The balance equations

$$
\varphi_{k}\left(a_{k}\right)=\theta_{k}\left(a_{k}\right)-\sum_{l} \int_{S_{l}} d a_{l} \gamma_{k l}\left(a_{k}, a_{l}\right) \theta_{l}\left(a_{l}\right)
$$

allow the calculation of all unknown $\varphi_{k}$ 's and $\theta_{k}$ 's. Doing an expansion as in Eq. (1) gives

$$
\varphi_{k}^{r}=\theta_{k}^{r}-\sum_{l=1}^{N} \sum_{s=0}^{P} \sqrt{\frac{S_{l}}{S_{k}}} M_{k l}^{r s} \theta_{l}^{s}
$$

This is a set of $N(P+1)$ equations in as many unknowns, some unknowns being $\theta$ 's and others $\varphi$ 's. The solution of these equations is straightforward.

5. Biases. At the end of the third section we discussed bias due to correlation between MC estimates of a quantity and of its variance. This bias can be avoided using independent MC estimates of the quantity and the variance, for example by breaking a run into two subruns and using the quantities $(\hat{M}$ s) estimated in one with the variances ( $\hat{\omega}$ 's) of the other.

Another source of bias arises because the solution of a set of simultaneous equa- 
tions with stochastic coefficients is biased away from the solution of the equations with the expected value of the coefficients. To see this, suppose $A=A_{0}+A_{1}$ is a nonsingular matrix whose expected value (entry by entry) is $A_{0}$ but which has a stochastic part $A_{1}$ with zero expectation value. Similarly, let $v=v_{0}+v_{1}$ be a vector whose expectation value is $v_{0}$, term by term and let $u$ be an unknown vector. We wish to compare the expectation value of the solution of

$$
A u=v
$$

with the solution $u_{0}$ to

$$
A_{0} u_{0}=v_{0}
$$

We have

$$
u_{0}=A_{0}^{-1} v_{0}
$$

and

$$
A_{0}^{-1}\left(A_{0}+A_{1}\right) u=A_{0}^{-1}\left(v_{0}+v_{1}\right) .
$$

Subtracting gives

$$
u-u_{0}+A_{0}^{-1} A_{1} u=A_{0}^{-1} v_{1},
$$

or since the expectation of $v_{1}$ and $A_{1}$ are zero

$$
\left\langle u-u_{0}\right\rangle=-A_{0}^{-1}\left\langle A_{1}\left(u-u_{0}\right)\right\rangle .
$$

Inserting (19) gives, to lowest nonzero order,

$$
\left\langle u-u_{0}\right\rangle=-A_{0}^{-1}\left\{\left\langle A_{1} A_{0}^{-1} v_{1}\right\rangle-\left\langle A_{1} A_{0}^{-1} A_{1} u_{0}\right\} .\right.
$$

A bias from this cause will appear both due to the least squares adjustment of the $\hat{M}$ 's and from the solution of the flow equations for the $\theta$ 's and $\varphi$ 's. The error, being quadratic in the stochastic quantities, should be proportional to $N_{0}^{-1}$.

We find the bias to be undetectably small in ordinary running. To verify its existence we did a long series of runs for a hollow cylinder, with diffusely reflecting walls, of length to radius ratio $L / R=5$, using four zones altogether: two on the cylinder wall and one for each end. The exact transmission probability for this cylinder (see next section and compare also Berman [11]) is 0.310525. Table I gives the results. They are consistent with a bias in the transmission of $-1.1 N_{0}^{-1}$ or $0.3 \%$ at $N_{0}$ $=10^{3}$. This is insignificant. Furthermore, we believe most of the small error can be eliminated by extrapolation to $N_{0}=\infty$ using correlated runs. To do this, we would use the same random numbers to generate sets of runs with two values of $N_{0}$. Then, assuming the error proportional to $N_{0}^{-1}$, we could extrapolate to $N_{0}^{-1}=0$.

We expect a third source of bias to occur because of truncation of the polynomial series. It should arise in those situations where the higher order polynomials are important. However, in our experience, the contributions due to higher order polynomials are generally small, and we have seen no evidence for this bias, even in runs with large numbers of particles. 
TABLE I

$\mathrm{L} / \mathrm{R}=5$ CYLINDER TRANSMISSION VS. NUMBER OF PARTICLES STARTED PER ZONE

\begin{tabular}{|c|c|c|c|c|}
\hline $10^{-3} \mathrm{~N}_{\mathrm{O}}$ & $\underline{n^{a}}$ & Transmission $^{\mathrm{b}}$ & Std. Dev. of Mean & $10^{5} \times$ Bias \\
\hline 40 & 9 & 0.310569 & 0.00037 & $4 \pm 37$ \\
\hline 20 & 11 & 0.310196 & 0.00038 & $-32 \pm 38$ \\
\hline 10 & 11 & 0.310612 & 0.0010 & $+9 \pm 100$ \\
\hline 5.2 & 11 & 0.310356 & 0.0011 & $-17 \pm 110$ \\
\hline 2 & 1000 & 0.309888 & 0.00017 & $-63 \pm 17$ \\
\hline 1 & 1000 & 0.309713 & 0.00025 & $-81 \pm 25$ \\
\hline
\end{tabular}

6. Results. We have run several geometries, including some run by Davis. For some of these an exact (nonstochastic) answer can be obtained by the numerical solution of an integral equation. In almost all cases, we have repeated each run with several random number chains, so that we have not only the mean answer but its standard deviation as well. We calculate a figure of merit by comparing the labor required for a given accuracy by our method to that required in a calculation like Davis' where particles are followed from injection until they emerge at either the source or the sink end. Since the labor is essentially proportional to the number of collisions, it is the ratio of the number of collisions required that we compare.

In the Davis type run, the variance would be

$$
\sigma_{D}^{2}=P(1-P) / N_{D}
$$

where $P$ is the transmission probability and $N_{D}$ the number of particles started. Most of our runs have had a unit source at one end, a perfect sink at the other, and have had, in addition to cylindrical symmetry, reflection symmetry about the mid-plane. Then the collision density with the wall will be one half the source plus an odd function of displacement from the symmetry point so that the number of collisions is $N_{D}$ times the wall area divided by twice the source area. Thus the labor required for a given accuracy in the Davis calculation can be predicted without running a problem.

Table II gives results for a number of simple cylindrical problems. The table shows that the figure of merit is always greater than 1 , and frequently 5-20. Using only $P^{0}$ is clearly inadequate. Most of the benefit from the expansion is obtained with $P^{1}$ and succeeding polynomials help only a little. Note that the runs which compare results for different orders of polynomial expansion are not independent in that the same MC run is used for estimating the coefficients of all orders. Also, it appears that very coarse zoning leads to poor results.

Table III describes results for three other geometries we have run. Here there is no exact solution. The figures of merit are again substantial, and again we find that using constant collision densities is inadequate and that the linear polynomial fit gives most of the improvement. However, because of the more complex geometry, higher polynomials now make a statistically significant contribution. 


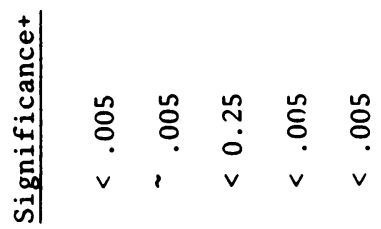

$$
\begin{aligned}
& \text { ๕ัญ ำ }
\end{aligned}
$$

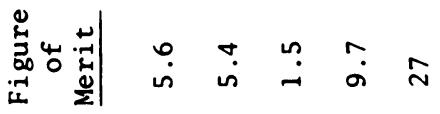

$$
\begin{aligned}
& \stackrel{\circ}{\circ}
\end{aligned}
$$

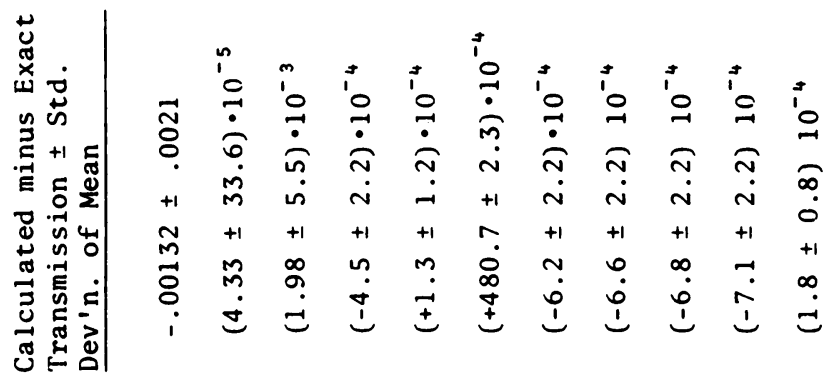

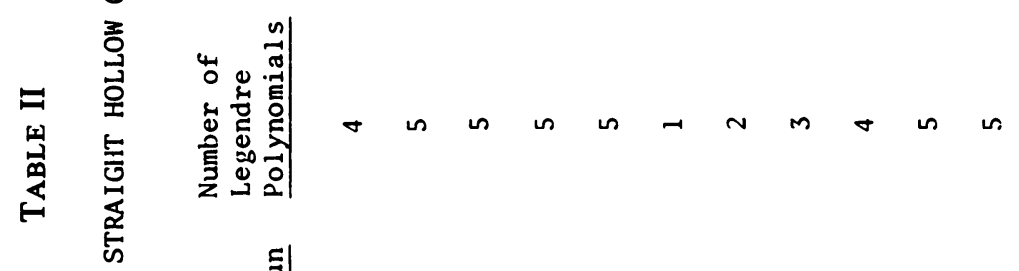

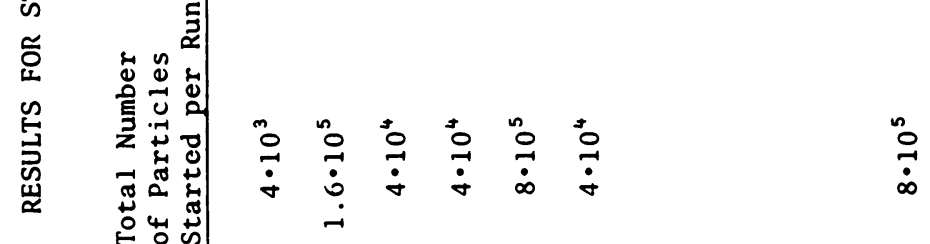

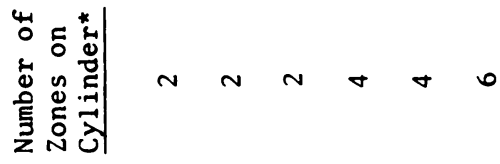

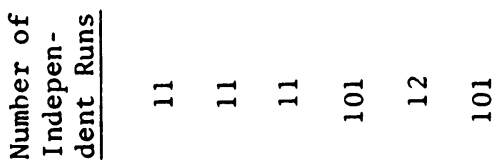

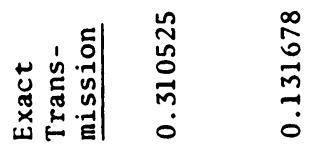

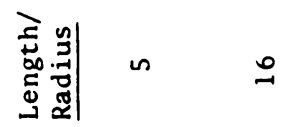




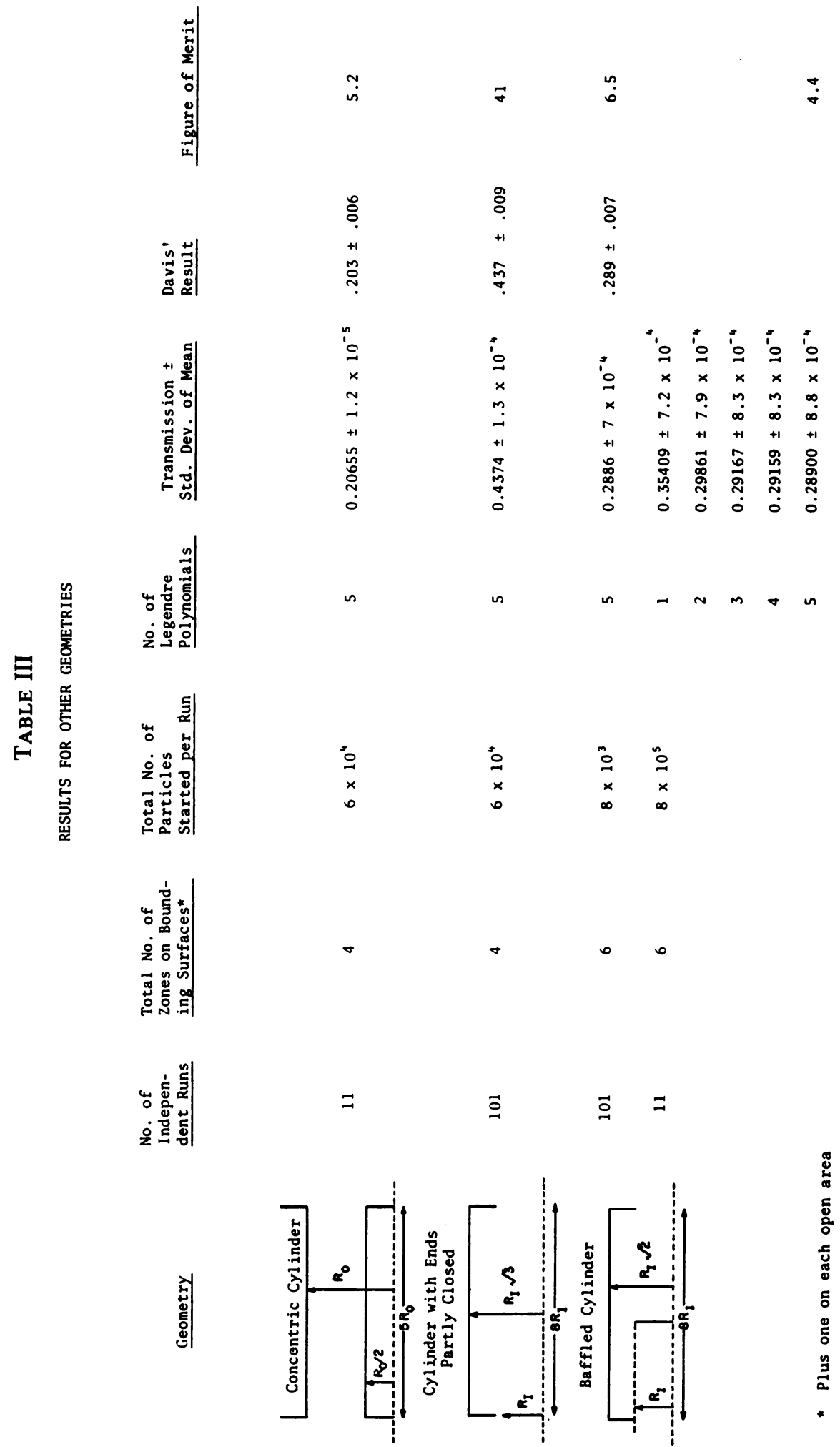


LOSSY CYLINDER

$(L / R=5)$

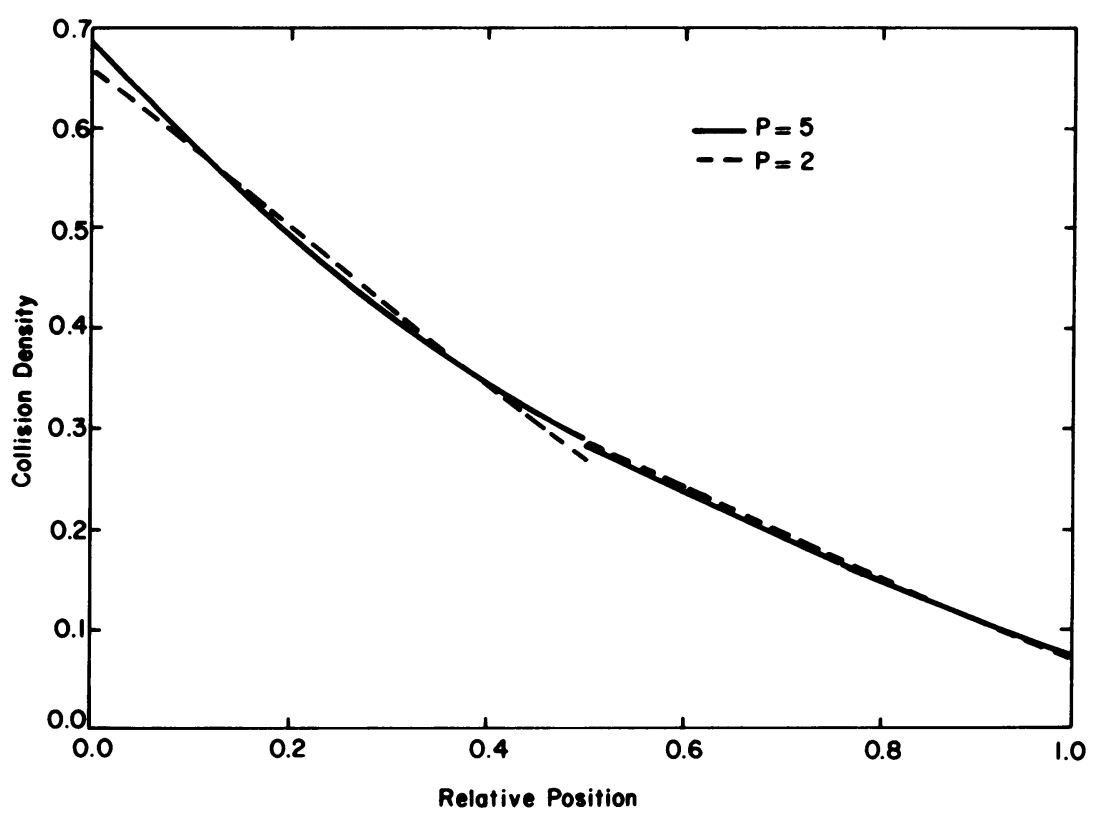

FIGURE 1

Figure 1 deals with a cylinder whose wall reflects only 10/11 of the incident particles. The curvature of the collision density is apparent. The $P^{0}+P^{1}$ polynomial fit now misses substantially. The transmissions are: Exact $0.18681, \theta^{0} P^{0}+\theta^{1} P^{1}$ (i.e., 2 polynomials) $0.18673,3$ or more polynomials 0.18603 . While $P^{2}$ does not make an important contribution to the transmission, it does improve the calculated collision density. Figure 2 shows the deviation of our calculation from the exact calculation for various polynomial fits. We have observed that the coefficients of the higher order polynomials $(P \gtrsim 3)$ are not well established by the Monte Carlo calculations with $4 \times 10^{5}$ total particles, and this lack of definition is evident in Figure 2.

7. Conclusions. We have demonstrated a MC calculation of Knudsen flow in cylindrically symmetric channels which is significantly more efficient of computer time than more straightforward calculations. A bias that is theoretically expected turns out to be very small and is not ordinarily detected. We have also discussed how to remove the bias. Further possible modifications include using some analytic view factors together with some estimated by MC or to select view factors from a library previously calculated. The results presented here have been for free flow in vacuum enclosures. However, the method has also been applied to cases where the enclosure contains a conservative scattering medium. In such situations, analytic calculation of the view factors is not generally possible. 


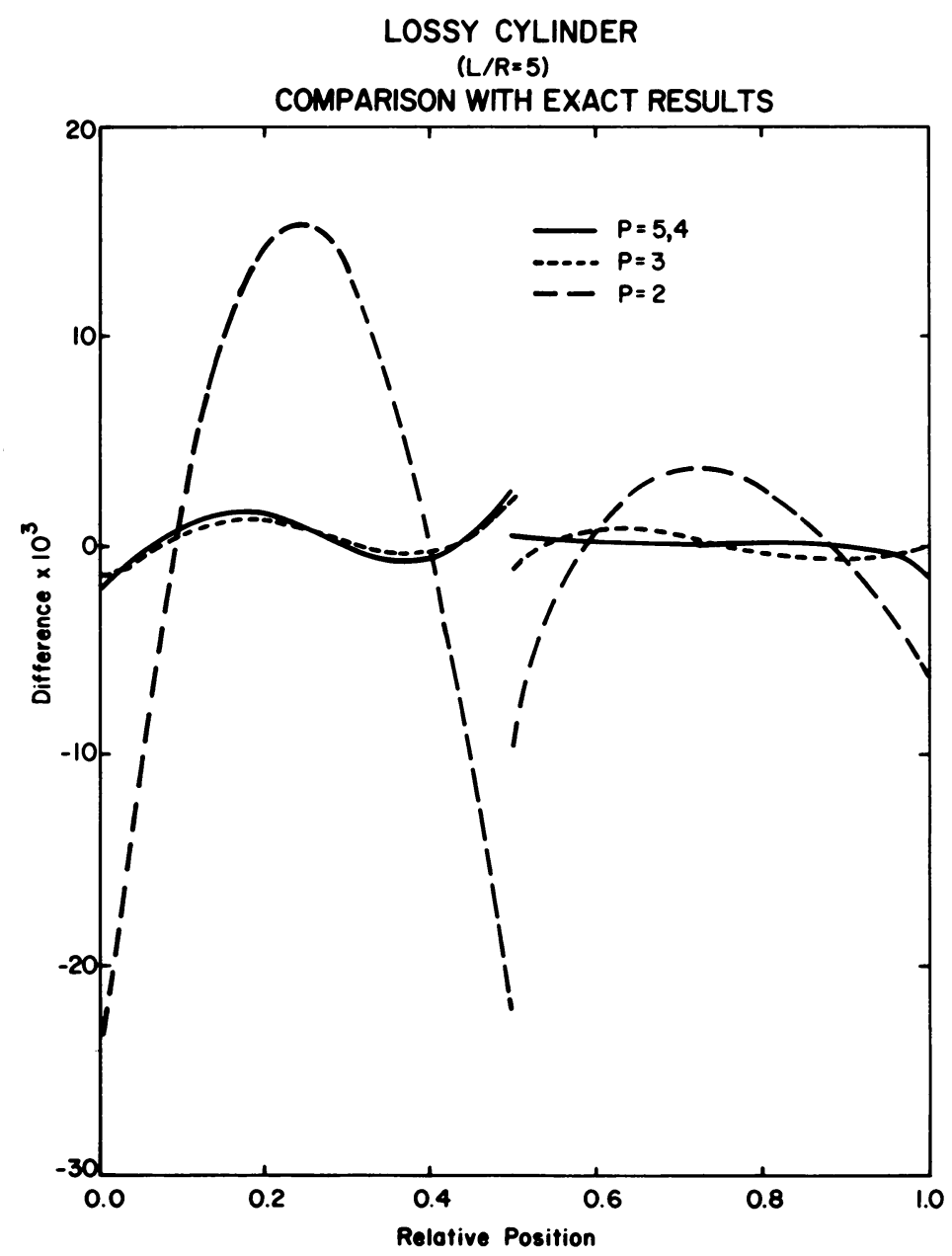

FIGURE 2

Acknowledgement. The present work is an expansion upon an original idea of B. E. Freeman, and much of the original coding was done by W. M. Taylor, A. R. Larson and L. G. Margolin. The authors are indebted to these people for their contributions.

University of California

Los Alamos Scientific Laboratory

Los Alamos, New Mexico 87545

1. D. H. DAVIS, "Monte Carlo calculation of molecular flow rates through a cylindrical elbow and pipes of other shapes," J. Appl. Phys, v. 31, 1960, pp. 1169-1176.

2. A. G. PETSCHEK, R. E. WILLIAMSON \& W. J. KRAUSER, "A novel Monte Carlo binning technique," Trans. Amer. Nuclear Soc., v. 27, 1977, pp. 377-378.

3. E. GARELIS \& T. E. WAINWRIGHT, "Free molecular flow in a right circular cylinder," Phys. Fluids, v. 16, 1973, pp. 476-481.

4. J. A. FLECK, JR., "Effective fluorescent lifetimes in ruby laser rods," J. Appl. Phys, v. 36, 1965 , pp. $1301-1306$.

5. D. BLECHSCHMIDT, "Monte Carlo study of light transmission through a cylindrical tube," J. Vac. Sci. T., v. 11, 1974, pp. 570-574. 
6. I. BERCEANU \& V. K. IGNATOVICH, "Molecular flow of ultracold neutrons through long tubes," Vacuum, v. 23, 1973, pp. $441-445$.

7. R. SIEGEL \& J. R. HOWELL, Thermal Radiation Heat Transfer, McGraw-Hill, New York, 1972.

8. J. M. HAMMERSLY \& D. C. HANDSCOMB, Monte Carlo Methods, Methuen, London, 1964.

9. T. M. JORDON, "Advanced Monte Carlo concepts in radiation shielding calculations: Methods and applications," Nuclear Engin. Design, v. 13, 1970, pp. 415-422.

10. B. L. BEERS \& V. W. PINE, "Functional expansion Monte Carlo electron transport calculations," IEEE Trans. Nuclear Sci, v. NS-23, 1976, pp. 1850-1856.

11. A. S. BERMAN, "Free molecule transmission probabilities," J. Appl. Phys, v. 36, 1965, p. 3356. 